\title{
Human Meniscus: From Biology to Tissue Engineering Strategies
}

\author{
Hélder Pereira ${ }^{a, b, c, d_{*}}$, Ibrahim Fatih Cengiz $z^{a, b}$, Joana Silva-Correia ${ }^{a, b}$, Joaquim Miguel Oliveira ${ }^{a, b}$, Rui Luís Reis ${ }^{a, b}$ and \\ João Espregueira-Mendes ${ }^{\text {a,b,c,d }}$ \\ 3B's Research Group - Biomaterials, Biodegradables and Biomimetics, Headquarters of the European Institute of \\ Excellence on Tissue Engineering and Regenerative Medicine, University of Minho, Taipas, Guimarães, Portugal \\ 'ICVS/3B's - PT Government Associated Laboratory, Guimarães, Portugal \\ ${ }^{\mathrm{C}}$ Clínica Espregueira-Mendes F.C. Porto Stadium - FIFA Medical Centre of Excellence, Porto, Portugal \\ ${ }^{d}$ Orthopedic Department, Centro Hospitalar Póvoa de Varzim, Vila do Conde, Portugal
}

\begin{abstract}
Once meniscus is damaged, a cascade of events occurs leading to degenerative joint changes of the knee. The morbidity of patients can significantly increase overtime and degeneration of the cartilage can progress, resulting in arthritis. Possibilities for treatment of meniscus lesions are primordially focused in repair and replacement (e.g., acellular scaffolds and meniscus allograft transplantation). Tissue Engineering and Regenerative Medicine have been providing new options in medical practice. However, these disciplines require deep understanding of the target tissue and physiopathology of the implicated disorder. In order to overcome the current limitations, fundamental studies have been made for developing reliable strategies aiming to obtain superior tissue healing. Herein, it is presented the most relevant insights and research directions on the fundamental biology and biomechanics of meniscus. The principles of tissue engineering (triad) and the significant in vitro and in vivo reports addressing meniscus regeneration are included, once these will provide the basements for future clinical directions.
\end{abstract}

\section{Introduction}

Meniscus is a complex tissue with an autonomous repair capability that is mostly restricted to the vascularized region, in the stable knee (Pereira et al. 2013c). Also for this fact, it still subsists as one of the most frequent injuries leading to orthopedic surgery (Garrett et al. 2006), thus causing work absenteeism and, in some cases, sports invalidity.

In the last few years, meniscal replacement/repair and regeneration are gaining increased attention (Lubowitz and Poehling 2011) as an alternative to meniscectomy. This is mainly due to the longterm functional instability of the knee and appearing of early osteoarthritis (Fayard et al. 2010) as a consequence of meniscus removal (Fig. 1).

Meniscus lesions treatment possibilities are still limited and quite often not very effective. This is not only due to the recent interest in its preservation but more importantly due to the fact that meniscus is poorly studied as compared to other tissues, in respect to: biomechanics, vascularity, innervation, cellularity, and extracellular matrix (ECM) composition.

Replacement of meniscus can be both total and partial (Monllau et al. 2010) and comprehends the use of cadaveric tissue. Repair strategies are almost restricted to suturing techniques, and its application greatly dependent on healing prognosis of meniscus which by its turn is dictated by

*Email: heldermdpereira@gmail.com 